\title{
Predictors of delayed failure of structural kyphoplasty for pathological compression fractures in cancer patients
}

\author{
Gary Rajah, MD, 1 David Altshuler, BS, ${ }^{2}$ Omar Sadiq, BS, ${ }^{2}$ V. Kwasi Nyame, MD, ${ }^{1}$ \\ Hazem Eltahawy, MD, PhD,' and Nicholas Szerlip, MD' \\ 1Department of Neurosurgery and ${ }^{2}$ School of Medicine, Wayne State University, Detroit, Michigan
}

\begin{abstract}
OBJECT Pathological compression fractures in cancer patients cause significant pain and disability. Spinal metastases affect quality of life near the end of life and may require multiple procedures, including medical palliative care and open surgical decompression and fixation. An increasingly popular minimally invasive technique to treat metastatic instabilities is kyphoplasty. Even though it may alleviate pain due to pathological fractures, it may fail. However, delayed kyphoplasty failures with retropulsed cement and neural element compression have not been well reported. Such failures necessitate open surgical decompression and stabilization, and cement inserted during the kyphoplasty complicates salvage surgeries in patients with a disease-burdened spine. The authors sought to examine the incidence of delayed failure of structural kyphoplasty in a series of cement augmentations for pathological compression fractures. The goal was to identify risk predictors by analyzing patient and disease characteristics to reduce kyphoplasty failure and to prevent excessive surgical procedures at the end of life.
\end{abstract}

METHODS The authors retrospectively reviewed the records of all patients with metastatic cancer from 2010 to 2013 who had undergone a procedure involving cement augmentation for a pathological compression fracture at their institution. The authors examined the characteristics of the patients, diseases, and radiographic fractures.

RESULTS In total, 37 patients underwent cement augmentation in 75 spinal levels during 45 surgeries. Four patients had delayed structural kyphoplasty failure necessitating surgical decompression and fusion. The mean time to kyphoplasty failure was $2.88 \pm 1.24$ months. The mean loss of vertebral body height was $16 \%$ in the patients in whom kyphoplasty failed and $32 \%$ in patients in whom kyphoplasty did not fail. No posterior intraoperative cement extravasation was observed in the patients in whom kyphoplasty had failed. The mean spinal instability neoplastic score was 10.8 in the patients in whom kyphoplasty failed and 10.1 in those in whom kyphoplasty did not fail. Approximately $50 \%$ of the kyphoplasty failures occurred at junctional spinal levels. All the patients in whom kyphoplasty failed had fractures in 3 or more cortical walls before treatment, whereas $46 \%$ of patients in the nonfailure group had fractures with breaching of 3 or more walls.

CONCLUSIONS Although rare, delayed failures of structural augmentation with cement during kyphoplasty do occur and can lead to additional surgeries. A possible predictive index may include wall integrity of the vertebral body, competency of the posterior tension band, and location of the kyphoplasty at a junctional spinal level. Additional studies are required to confirm these findings.

http://thejns.org/doi/abs/10.3171/2014.11.SPINE14909

KEY WORDS metastatic cancer; pathological fracture; kyphoplasty; oncology

$\mathrm{T}$ HE vertebral column is the primary osseous target of metastatic cancer. ${ }^{15}$ Approximately $30 \%$ of patients with neoplastic processes will develop symptomatic spinal metastasis. ${ }^{2}$ In such cases, pain will account for roughly $90 \%$ of presenting symptoms followed by neurological deficits in the lower extremity. ${ }^{9}$ Pathological compression fractures cause high morbidity rates, and analgesics used to treat bone pain exacerbate these high rates at the end of life. Introduced in the 1980s, percutaneous injection of cement into a vertebral body is used to treat vertebral hemangiomas ${ }^{6}$ and osteoporotic vertebral compression fractures. This procedure, called vertebroplasty, was later modified to yield kyphoplasty, consisting of the addition of an inflatable balloon to augment reduction of vertebral body fractures. ${ }^{7}$

Kyphoplasty effectively relieves acute pain due to path-

ABBREVIATION SINS = Spinal Instability Neoplastic Score.

SUBMITTED September 8, 2014. ACCEPTED November 25, 2014.

INCLUDE WHEN CITING Published online May 8, 2015; DOI: 10.3171/2014.11.SPINE14909.

DISCLOSURE The authors report no conflict of interest concerning the materials or methods used in this study or the findings specified in this paper. 
ological fractures in cancer patients and due to osteoporosis. For example, $84 \%$ of patients reported marked or complete relief of preoperative pain after a kyphoplasty. ${ }^{5}$ In addition, kyphoplasty may reduce analgesic use, further helping to improve the quality of life of cancer patients. ${ }^{1}$ Complications of this procedure are relatively rare, ${ }^{5}$ with extravasation of cement, cord compression, and neurological deficits being the most serious complications. ${ }^{12}$ Fractures at adjacent segments have also been extensively reported, especially in patients with osteoporosis. In our practice, we commonly use kyphoplasty as a palliative modality to treat pathological compression fractures in cancer patients. The method is minimally invasive and causes little or no interruption of cancer therapy.

We observed delayed retropulsion of cement after kyphoplasty, indicating delayed failure of kyphoplasty-treated fractures, and these failures necessitated surgery. Given that kyphoplasty is a palliative approach in metastatic cancer patients, great care must be taken to ensure no further morbidity is created. Being able to determine predictors of kyphoplasty failure will help stratify the risk of this procedure for cancer patients. We set out to determine the incidence of this delayed structural failure in our patients to identify its risk factors. To the best of our knowledge, this is the first report examining delayed kyphoplasty failures in cancer patients.

\section{Methods}

\section{Data Collection and Spine Assessment}

We retrospectively reviewed the records of all metastatic cancer patients treated at our institution from 2010 to 2013. Kyphoplasty was performed during 45 surgeries in 75 spinal levels in 37 patients. All procedures were performed using the Kyphon Kyphoplasty kit (Medtronic). Multivariate statistical analysis was performed with Microsoft Excel and SPSS software. All procedures were carried out by the Wayne State University Department of Neurosurgery staff, specifically, by Drs. Szerlip and Eltahawy. Survival data were obtained using the Social Security Death Index or last documented encounter entered into the electronic medical record of a patient. Kyphoplasty failure was defined as delayed retropulsion of cement into the spinal canal requiring operative spinal decompression and fusion.

We also included the Spinal Instability Neoplastic Score (SINS) in our analysis to design a screening procedure that identifies patients at risk for kyphoplasty failure. The SINS is used to categorize the degree of spinal instability in patients. Its scoring includes location of the metastatic tumor, presence of pain, the type of bone lesion, spinal alignment, the degree of collapse of the vertebral body, and involvement of posterolateral elements in the tumor. A spine with a SINS of 0-6 was defined as stable, of 7-12 as potentially unstable, and of $13-18$ as unstable. ${ }^{4}$ Multiple reviewers assessed cortical breaches by examining the sagittal reconstructions on preoperative CT scans and graded the breaches on a scale of $0-4$, depending on the number of cortical walls that had a break. Cement extravasation was assessed by reviewing intraoperative fluoroscopy data. Data are reported as mean $\pm \mathrm{SD}$.

\section{Procedure}

We used a standard transpedicular approach when possible and Jamshidi needles and bone fillers under anteroposterior and lateral fluoroscopy views with shots taken at 0.5 -ml acrylate incremental injections.

\section{Results}

Kyphoplasty procedures at 75 spinal levels were performed during 45 surgeries in 37 patients, $61 \%$ of whom were female. Of these procedures, 55\% were performed in the thoracic spine and $45 \%$ in the lumbar spine. More than $50 \%$ of the metastatic tumors had originated from multiple myelomas and lung carcinomas (Table 1). In 4 patients (corresponding to $5.3 \%$ of all kyphoplasty procedures), delayed kyphoplasty failure met our criterion of structural failure requiring operative decompression and stabilization. Such failures occurred on average $2.88 \pm$ 1.24 months after the kyphoplasty. The mean age was 52 years for the patients in whom kyphoplasty failed and 60 years for patients in whom kyphoplasty did not fail (Table 1). No significant differences in age, sex, tumor type (the 4 kyphoplasty failures affected patients with neck adenocarcinoma or with cancer of the breast, lung, or colon), cement volume (mean $3.6 \mathrm{ml}$ ), or level (2 thoracic-level failures at T-5 and T-6, and 2 lumbar-level failures at L-1) were observed.

Overall, $80 \%$ of the patients reported improvement of their pain postoperatively (Table 2). Among the patients in the kyphoplasty-failure group, 50\% had a fractured anterior vertebral body wall, and $75 \%$ had a fractured posterior vertebral body wall; the corresponding rates were

TABLE 1. Demographics and type of primary cancer of the patients who underwent kyphoplasty and in whom the procedure failed or did not fail ${ }^{*}$

\begin{tabular}{lcc}
\hline \multirow{2}{*}{ Variable } & \multicolumn{2}{c}{ Kyphoplasty } \\
\cline { 2 - 3 } & Failure & No Failure \\
\hline Mean age at treatment, yrs (SD) & $52.5(7.3)$ & $60.1(1.5)$ \\
\hline Surgeries & 4 & 41 \\
\hline Female sex & $3(75.0)$ & $25(61.0)$ \\
\hline Cancer type & & \\
\hline Lung & $1(25.0)$ & $8(19.5)$ \\
\hline Breast & $1(25.0)$ & $5(12.2)$ \\
\hline Colon & $1(25.0)$ & $0(0.0)$ \\
\hline Neck adenocarcinoma & $1(25.0)$ & $0(0.0)$ \\
\hline Prostate & $0(0.0)$ & $1(2.4)$ \\
\hline Renal cell & $0(0.0)$ & $1(2.4)$ \\
\hline Multiple myeloma & $0(0.0)$ & $21(51.2)$ \\
\hline Polycythemia & $0(0.0)$ & $2(4.8)$ \\
\hline NHL & $0(0.0)$ & $1(2.4)$ \\
\hline CML & $0(0.0)$ & $1(2.4)$ \\
\hline Lymphoma & $0(0.0)$ & $1(2.4)$ \\
\hline Mean length of survival, mos (SD) & $6.3(2.1)$ & $17.7(1.8)$ \\
\hline
\end{tabular}

$\mathrm{CML}=$ chronic myeloid leukemia; NHL = non-Hodgkin lymphoma.

* Values represent the number of patients (\%), unless indicated otherwise. 
TABLE 2. Characteristics and outcomes in the 2 patient groups in this study*

\begin{tabular}{|c|c|c|}
\hline \multirow[b]{2}{*}{ Variable } & \multicolumn{2}{|c|}{ Kyphoplasty } \\
\hline & Failure & No Failure \\
\hline \multicolumn{3}{|l|}{ Spinal level } \\
\hline Thoracic & 2 & 39 \\
\hline Lumbar & 2 & 32 \\
\hline \multicolumn{3}{|l|}{ Postop pain procedures $†$} \\
\hline Same & $1(25.0)$ & $7(17.1)$ \\
\hline Better & $3(75.0)$ & $33(80.5)$ \\
\hline Worse & $0(0.0)$ & $1(2.4)$ \\
\hline $\begin{array}{l}\text { Mean height loss of vertebral body collapse, } \\
\% \text { (SD) }\end{array}$ & $16.5(7.7)$ & $32.0(2.5)$ \\
\hline \multicolumn{3}{|l|}{ Spinal alignment procedures (\%) } \\
\hline Normal alignment & $1(25.0)$ & $8(19.5)$ \\
\hline De novo deformity & $1(25.0)$ & $5(12.2)$ \\
\hline Subluxation/translation deformity & $1(25.0)$ & $0(0.0)$ \\
\hline Fractures in $\geq 3$ cortical walls, no. of pts (\%) & $4(100.0)$ & $19(46.3)$ \\
\hline \multicolumn{3}{|l|}{ Cement extravasation } \\
\hline No & $3(75.0)$ & $62(88.0)$ \\
\hline Yes & $1(25.0)$ & $9(12.7)$ \\
\hline Mean SINS (SD) & $10.8(0.8)$ & $10.1(0.2)$ \\
\hline \multicolumn{3}{|l|}{ SINS class } \\
\hline Stable & $0(0.0)$ & $2(2.8)$ \\
\hline Potentially unstable & $3(75.0)$ & $58(81.7)$ \\
\hline Unstable & $1(25.0)$ & $11(15.5)$ \\
\hline
\end{tabular}

pts = patients.

* Values represent the number of kyphoplasty procedures (\%), unless indicated otherwise.

$\dagger$ The change in postoperative pain was assessed relative to preoperative pain.

$20 \%$ and $60 \%$, respectively, in the patients in the nonfailure group. All patients in whom kyphoplasty failed had 3 or more vertebral wall fractures, while this fracture extent was observed in $46 \%$ of those in whom kyphoplasty did not fail ( $\mathrm{p}<0.05$, Fisher's exact test) (Table 2). One patient in the kyphoplasty-failure group had a preexisting kyphotic deformity. The odds of having kyphoplasty failure was 24.2 times greater in patients with 3 or more wall fractures than in those without these failures (95\% CI 1.25-471.2, $p=0.035$ ). Height loss of the vertebral body was $16 \%$ in the kyphoplasty-failure group and $32 \%$ in the nonfailure group (Table 2). Interestingly, we observed minimal cement extravasation during the procedure in $1(25.0 \%)$ of the levels in which kyphoplasty failed, and in $9(12.7 \%)$ of the levels in the nonfailure group, with only 1 patient having posterior extravasation.

Among the patients in whom kyphoplasty failed, 50\% had bilateral involvement of metastatic posterolateral elements, which was observed in only $12 \%$ of patients in the nonfailure group. Mean SINSs were 10.8 for the kyphoplasty-failure group and 10.1 for the nonfailure group $(\mathrm{p}$ $>0.05$ ) According to the SINS classification, the kyphoplasty-failure group included 3 patients (75\%) in whom the spine was classified as potentially unstable, and the spine in (25\%) 1 patient was classified as unstable. A roughly similar distribution of spinal instability was seen in the nonfailure group. The mean length of survival was 6.3 \pm 2.1 months for the patients in the kyphoplasty-failure group and $17.7 \pm 1.8$ months for the patients in the nonfailure group (Table 1). Among the patients in whom kyphoplasty had failed, $1(25 \%)$ was alive at the last follow-up 18 months after the kyphoplasty, compared with $26(63 \%)$ of the patients in the nonfailure group.

Figure 1 shows pre- and intraoperative spinal images, and representative images after kyphoplasty failure in the 4 patients in whom kyphoplasty failed. Preoperatively, 2 of the 4 patients had large lucent areas within the vertebral body, with a very thin cortical bone. All 4 patients in the kyphoplasty-failure group initially presented with recurrence of preoperative pain that had resolved after the first procedure. One patient also had weakness in the lower extremity.

\section{Discussion}

Our results indicate that kyphoplasty-failure rates in metastatic disease are much higher than previously reported in similar studies of patients with metastatic cancer ${ }^{5}$ or osteoporosis. ${ }^{16}$ The failure rate observed probably resulted from a combination of local disease progression and catastrophic structural failure of the vertebral body due to

\section{Preop Intraop Failures}

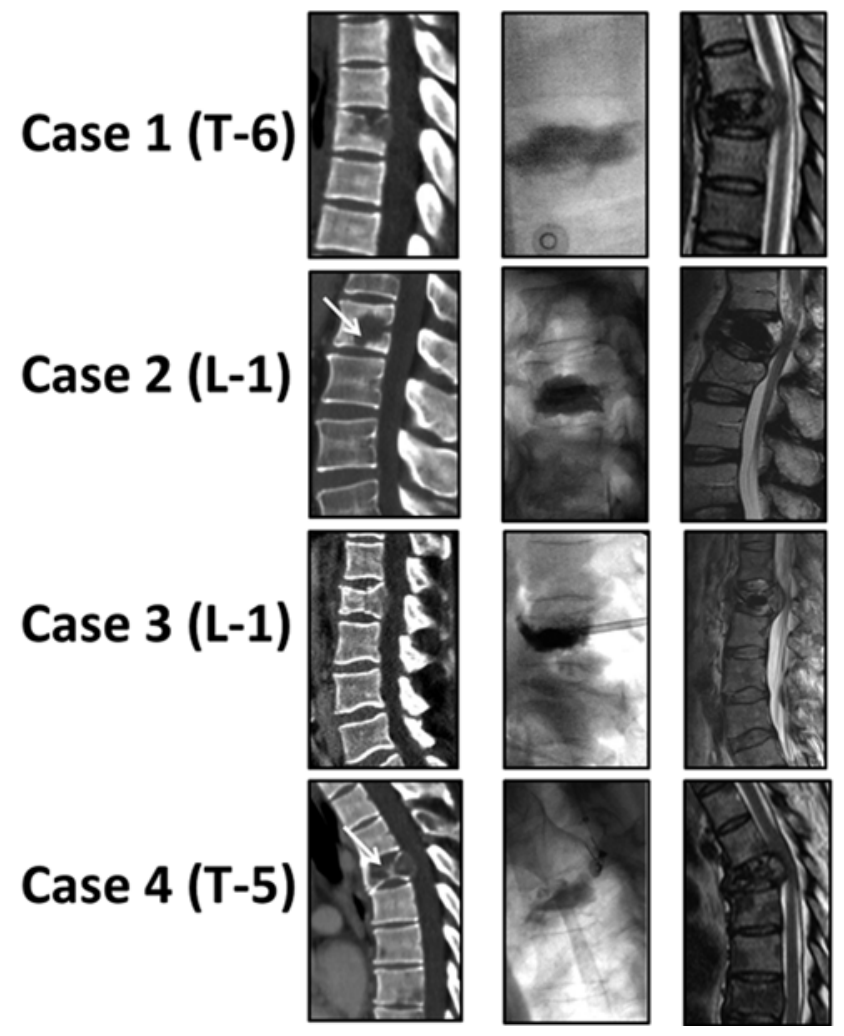

FIG. 1. Spinal images taken preoperatively, intraoperatively, and at the time of kyphoplasty failure in the 4 cases of delayed kyphoplasty failure. Note the large cortical defects and lucencies marked by the white arrows on preoperative CT scans for Cases 2 and 4. 
structural instability. We did not find previously published reports on delayed kyphoplasty failure.

Many studies have reported spinal cord compression at the time of the procedure resulting from dorsal cement extravasation. One recent study of 97 kyphoplasty procedures for metastatic disease reported that none of the patients required a return to the operating room for cord compression due to cement dislodgement and noted a cement extravasation rate of $9 \% .{ }^{5}$ One large meta-analysis including both osteoporotic and metastatic lesions found a cement extravasation rate of $8.6 \%$ (193 out of 2239 cases) and a spinal cord compression rate of $0.2 \%$ ( 1 out of 431 cases). ${ }^{14}$ Moreover, a systematic review identified 8 case reports out of 168 reviewed studies reporting posterior cement extravasation requiring operative decompression. ${ }^{3}$

Only 1 of these 8 reports mentioned that spinal cord compression was initially performed to address metastatic cancer. In this report, a 50-year-old woman with a T-1 pathological compression fracture from metastatic breast cancer had intraoperative cement extravasation and developed weakness over a 48 -hour postoperative period. She was taken back to the operating room for a decompression. ${ }^{12}$ This observation is in contrast to those in our study in which kyphoplasty failure was delayed on average by 2-3 months. In fact, none of the patients with delayed kyphoplasty failure in our series had any posterior extravasation of cement as monitored with intraoperative fluoroscopy, and none needed to return to the operating room immediately after the procedure.

Currently, kyphoplasty is being combined with other modalities, including radiotherapy to the kyphoplasty level, and these combined approaches resolve pain well; ${ }^{8}$ however, these approaches require monitoring for any possible effects on delayed kyphoplasty failure. Radiated vertebral bodies have been shown to have a higher rate of fracture progression, especially if the lesion is large and lytic. ${ }^{13}$ Among the 4 patients in our study in whom kyphoplasty failed, $2(50 \%)$ received radiation before kyphoplasty. One patient (25\%) in the kyphoplasty-failure group had preoperative chemotherapy. By comparison, in the nonfailure group, 5 (12.2\%) of the patients underwent radiotherapy before kyphoplasty, and 24 (58.5\%) underwent chemotherapy. Adjuvant radiation was recommended for any biopsies taken during the kyphoplasty procedure that indicated the presence of cancer cells. Salvage surgery consisted of posterolateral decompression and spinal fusion at the lumbar levels and minimally invasive surgery with a lateral extracavitary approach and fusion at the thoracic levels. All patients experienced improvement in their predecompression symptoms.

Predictors of kyphoplasty failure in our study included overall health of the vertebral body as measured by the integrity of the cortical walls. In addition, the SINS indicated that junctional levels may be more susceptible to failure; for example, $50 \%$ of the kyphoplasty failures in this study occurred at junctional levels (that is, at L-1). This finding was likely related to the increased mobility at these levels. Lastly, posterolateral element involvement in the metastasis was more frequent in the kyphoplasty-failure group. Posterolateral involvement because of the lack of a competent posterior tension band was likely respon- sible for decreased overall spinal stability with resultant increased load on the vertebral body.

Previous studies have reported cement extravasation as a risk factor for kyphoplasty failure; however, we noted only minimal cement extravasation in 1 of the cases in which kyphoplasty eventually failed. A large series of 190 cases of kyphoplasty performed in myeloma patients reported a cement extravasation rate of 5\%, with 1 patient requiring operative decompression..$^{10}$ This study reported a posterior defect in the vertebral body that had been missed because only a preoperative MRI scan was obtained, prompting the authors to recommend routine preoperative CT scanning for metastatic fractures. ${ }^{10}$ Lastly, a study of 99 cases of kyphoplasty in metastatic cancer patients reported a $12 \%$ cement extravasation rate, with no delayed failures over a 2-year follow-up period. ${ }^{11}$ This study, however, excluded patients with tumor involvement of the posterior cortex and spinal stenosis.

In our study, the patients in whom the kyphoplasty failed were younger and had shorter postoperative survival. Although we did not identify a causal relationship, this observation reinforces the idea that the lower survival could have resulted from a combination of disease progression with structural kyphoplasty failure. Our study sample was small, however, precluding any solid conclusions.

\section{Conclusions}

To the best of our knowledge, we report the first study of delayed kyphoplasty failure. We noted that $5 \%$ of kyphoplasty procedures in patients with spinal metastases showed delayed failure, which necessitated operative decompression. In addition, our report identified multiple possible predictors of delayed kyphoplasty failures. The most important risk factor appeared to be the overall integrity of the vertebral body. Others included the junctional levels, involvement of the posterolateral element in the disease, and previous radiotherapy. According to our experience, it appears that along with the trend toward minimizing extensive surgical interventions in metastatic disease of the vertebral column, we now offer kyphoplasty as treatment more liberally, occasionally approaching the limits of its indications. On the basis of these findings, we recommend caution when performing a kyphoplasty at junctional levels with multiple cortical defects. While additional randomized trials are necessary, we hope this study will aid in identifying patients at high risk for kyphoplasty failure and will help surgeons make informed decisions about which surgical intervention will provide the best risk-to-benefit ratio.

\section{References}

1. Berenson J, Pflugmacher R, Jarzem P, Zonder J, Schechtman K, Tillman JB, et al: Balloon kyphoplasty versus non-surgical fracture management for treatment of painful vertebral body compression fractures in patients with cancer: a multicentre, randomised controlled trial. Lancet Oncol 12:225-235, 2011

2. Cobb CA III, Leavens ME, Eckles N: Indications for nonoperative treatment of spinal cord compression due to breast cancer. J Neurosurg 47:653-658, 1977 
3. Eck JC, Nachtigall D, Humphreys SC, Hodges SD: Comparison of vertebroplasty and balloon kyphoplasty for treatment of vertebral compression fractures: a meta-analysis of the literature. Spine J 8:488-497, 2008

4. Fourney DR, Frangou EM, Ryken TC, Dipaola CP, Shaffrey CI, Berven SH, et al: Spinal instability neoplastic score: an analysis of reliability and validity from the spine oncology study group. J Clin Oncol 29:3072-3077, 2011

5. Fourney DR, Schomer DF, Nader R, Chlan-Fourney J, Suki D, Ahrar K, et al: Percutaneous vertebroplasty and kyphoplasty for painful vertebral body fractures in cancer patients. J Neurosurg 98 (1 Suppl):21-30, 2003

6. Galibert P, Deramond H, Rosat P, Le Gars D: [Preliminary note on the treatment of vertebral angioma by percutaneous acrylic vertebroplasty.] Neurochirurgie 33:166-168, 1987 (Fr)

7. Garfin SR, Yuan HA, Reiley MA: New technologies in spine: kyphoplasty and vertebroplasty for the treatment of painful osteoporotic compression fractures. Spine (Phila Pa 1976) 26:1511-1515, 2001

8. Gerszten PC, Germanwala A, Burton SA, Welch WC, Ozhasoglu C, Vogel WJ: Combination kyphoplasty and spinal radiosurgery: a new treatment paradigm for pathological fractures. J Neurosurg Spine 3:296-301, 2005

9. Gokaslan ZL, York JE, Walsh GL, McCutcheon IE, Lang FF, Putnam JB Jr, et al: Transthoracic vertebrectomy for metastatic spinal tumors. J Neurosurg 89:599-609, 1998

10. Huber FX, McArthur N, Tanner M, Gritzbach B, Schoierer O, Rothfischer W, et al: Kyphoplasty for patients with multiple myeloma is a safe surgical procedure: results from a large patient cohort. Clin Lymphoma Myeloma 9:375-380, 2009

11. Pflugmacher R, Taylor R, Agarwal A, Melcher I, Disch A, Haas NP, et al: Balloon kyphoplasty in the treatment of metastatic disease of the spine: a 2-year prospective evaluation. Eur Spine J 17:1042-1048, 2008

12. Ratliff J, Nguyen T, Heiss J: Root and spinal cord compres- sion from methylmethacrylate vertebroplasty. Spine (Phila Pa 1976) 26:E300-E302, 2001

13. Rose PS, Laufer I, Boland PJ, Hanover A, Bilsky MH, Yamada J, et al: Risk of fracture after single fraction image-guided intensity-modulated radiation therapy to spinal metastases. J Clin Oncol 27:5075-5079, 2009

14. Taylor RS, Fritzell P, Taylor RJ: Balloon kyphoplasty in the management of vertebral compression fractures: an updated systematic review and meta-analysis. Eur Spine J 16:10851100,2007

15. Toma CD, Dominkus M, Nedelcu T, Abdolvahab F, Assadian $\mathrm{O}$, Krepler P, et al: Metastatic bone disease: a 36-year single centre trend-analysis of patients admitted to a tertiary orthopaedic surgical department. J Surg Oncol 96:404-410, 2007

16. Wardlaw D, Cummings SR, Van Meirhaeghe J, Bastian L, Tillman JB, Ranstam J, et al: Efficacy and safety of balloon kyphoplasty compared with non-surgical care for vertebral compression fracture (FREE): a randomised controlled trial. Lancet 373:1016-1024, 2009

\section{Author Contributions}

Conception and design: Szerlip, Eltahawy. Acquisition of data: Szerlip, Rajah, Altshuler, Sadiq, Nyame. Analysis and interpretation of data: Szerlip, Altshuler. Drafting the article: Szerlip, Rajah. Critically revising the article: Szerlip, Rajah, Altshuler, Eltahawy. Reviewed submitted version of manuscript: all authors. Approved the final version of the manuscript on behalf of all authors: Szerlip. Statistical analysis: Szerlip, Altshuler, Sadiq. Study supervision: Szerlip.

\section{Correspondence}

Nicholas Szerlip, Department of Neurosurgery, Wayne State University, 4201 St. Antoine St., Ste. 6E, Detroit, MI 48201. email: nszerlip@med.wayne.edu. 